\title{
FEEDBACK STABILIZATION OF DISPLACED PERIODIC ORBITS: APPLICATION TO BINARY ASTEROID
}

\author{
Jules Simo* and Colin R. Mclnnes ${ }^{\dagger}$
}

\begin{abstract}
This paper investigates displaced periodic orbits at linear order in the circular restricted Earth-Moon system (CRTBP), where the third massless body utilizes a hybrid of solar sail and a solar electric propulsion (SEP). A feedback linearization control scheme is implemented to perform stabilization and trajectory tracking for the nonlinear system. Attention is now directed to binary asteroid systems as an application of the restricted problem. The idea of combining a solar sail with an SEP auxiliary system to obtain a hybrid sail system is important especially due to the challenges of performing complex trajectories.
\end{abstract}

\section{INTRODUCTION}

The design of spacecraft trajectories is a crucial task in space mission design. However, propellant usage is a critical parameter for any spacecraft mission, thus the choice of an efficient control strategy is important. Propellantless spacecraft propulsion systems such as solar sailing rely on solar radiation pressure, the flux of momentum transported by sunlight, to provide propulsive force. A solar sail is then a large, flat, lightweight reflective surface deployed in space that can propel spacecraft without the use of propellant. Although the force on a solar sail spacecraft is less than a conventional chemical rocket, the solar sail spacecraft constantly accelerates over time and achieves a significant energy change. Therefore, this form of propulsion can in principle provide energy changes greater than are possible with either ion or chemical propellants. This paper covers the results of a study on displaced periodic orbits in the Earth-Moon system in which the third body uses a hybrid solar sail. The hybrid sail model is composed of two low thrust propulsion systems, namely a solar sail and solar electric propulsion. In a prior study, displaced lunar orbits was investigated using low-thrust propulsion. 1,2,3,4,5,6,7 The idea of combining a solar sail with an auxiliary SEP system to obtain a hybrid sail system is important due to the challenges of performing complex missions. ${ }^{8,9,10}$ The solar electric propulsion system possesses high specific impulse $\left(I_{s p} \approx 3000 \mathrm{sec}\right)$. SEP consumes propellant and decreases the mass of the spacecraft, whereas the solar sail does not consume any propellant. This form of propulsion is useful for some high energy missions, but unlike solar sails, they have a finite $\Delta V$ capability, which makes them unsuitable for missions where a non-Keplerian orbit has to be maintained over indefinite periods of time.

Solar sails can also be utilised to maintain highly non-Keplerian orbits, such as closed orbits displaced high above the ecliptic plane. Solar sails are especially suited for such non-Keplerian orbits, ${ }^{11,12,13,14,15}$ since they can apply a propulsive force continuously over long periods. In such trajectories, a sail can be used as a communication satellite for high latitudes. For example, the orbital plane of the sail can be displaced above the orbital plane of the Earth, so that the sail can

\footnotetext{
*Academic Visitor, Department of Mechanical and Aerospace Engineering, University of Strathclyde, Glasgow, G1 1XJ, United Kingdom. Email: jules.simo@strath.ac.uk.

${ }^{\dagger}$ Professor, Department of Mechanical and Aerospace Engineering, University of Strathclyde, Glasgow, G1 1XJ, United Kingdom. Email: colin.mcinnes@strath.ac.uk.
} 
stay fixed above the Earth at some distance, if the orbital periods are equal. Displaced orbits have more recently been developed using collocation schemes and Finite-difference methods. ${ }^{16,17}$ A new concept of creating artificial equilibria above $L_{1}$ point in the Sun-Earth system for Earth observation has been proposed, in which the third body uses a hybrid of solar sail and SEP. These artificial equilibria have potential applications for future space physics and Earth observation missions. Hybrid low-thrust propulsion has been proposed on the same spacecraft to enable a pole-sitter orbit. ${ }^{18}$ Displaced geostationary orbits using hybrid low-thrust propulsion has also been proposed to increase the capacity of the geostationary ring. ${ }^{19}$ In fact, the pressure from sunlight reflecting off the solar sail pushes the satellite above or below geostationary orbit, and also displaces the centre of the orbit behind the Earth, away from the Sun.

The Earth-Moon libration points have been a topic of great interest in recent years. Particularly attractive are the orbits around the collinear points because their unique positions are advantageous for several important applications in space mission design. ${ }^{20,21,22,23,24,25,26}$ Such orbits cannot be

maintained without active control due to their instability. ${ }^{24,25,26}$ If the orbit maintains visibility from Earth, a spacecraft on it (near the $L_{2}$ point) can be used to provide communications between the equatorial regions of the Earth and the lunar poles. Moreover, if another communications satellite is located at the $L_{1}$ point, there could be continuous communications coverage between the equatorial region of the Earth and the entire lunar surface.

This paper investigates displaced periodic orbits at linear order in the circular restricted EarthMoon system, where the third massless body utilizes a hybrid of solar sail and a solar electric propulsion system. In particular, periodic orbits in the vicinity of the Lagrange points in the EarthMoon system will be explored along with their applications. Firstly we describe the dynamic model of the hybrid sail. The first-order approximation is derived for the linearized equations of motion. Then, a feedback linearization control scheme ${ }^{27}$ is proposed and implemented. The main idea of this approach is to cancel the nonlinearities and to impose desired linear dynamics satisfied by the solar sail. We then select the SEP control, which takes into consideration the nonlinearity cancellation and the stabilizing linear control. When the control is applied to the nonlinear system, asymtotic stability is achieved. This provides the key advantage that the displacement distance of the hybrid sail is then constant. A hybrid concept for displaced periodic orbits in the Earth-Moon system has been developed. In recent years a significant progress has been made in discovery of binary asteroids among all populations in the solar system. ${ }^{28}$ The binary near-Earth asteroids (NEAs) have been discovered from a combination of lightcurve and radar observations. Near-Earth Objects include asteroids and comets whose orbits approach or intersect the Earths orbit around the Sun. ${ }^{29,30,31}$ A natural extension of the hybrid concept is then to investigate the possible transition to a binary system. Finally, this research integrates results from the dynamics in order to develop a mission to a binary asteroid system. Therefore, important generalizations of the physical nature of the primaries lead to modified versions of the restricted problem, in which the primaries have either variable or equal masses. The displaced orbits found in the reference ${ }^{16}$ show large excursions in displacement distance. In practice, a constant displacement distance may lead to easier tracking from the lunar surface for communications applications. Finally, we evaluate the performance of the hybrid sail approach.

\section{SYSTEM MODEL}

In this work $m_{1}$ represents the larger primary (Earth), $m_{2}$ the smaller primary (Moon) and we will be concerned with the motion of a hybrid sail that has negligible mass. It is always assumed that 


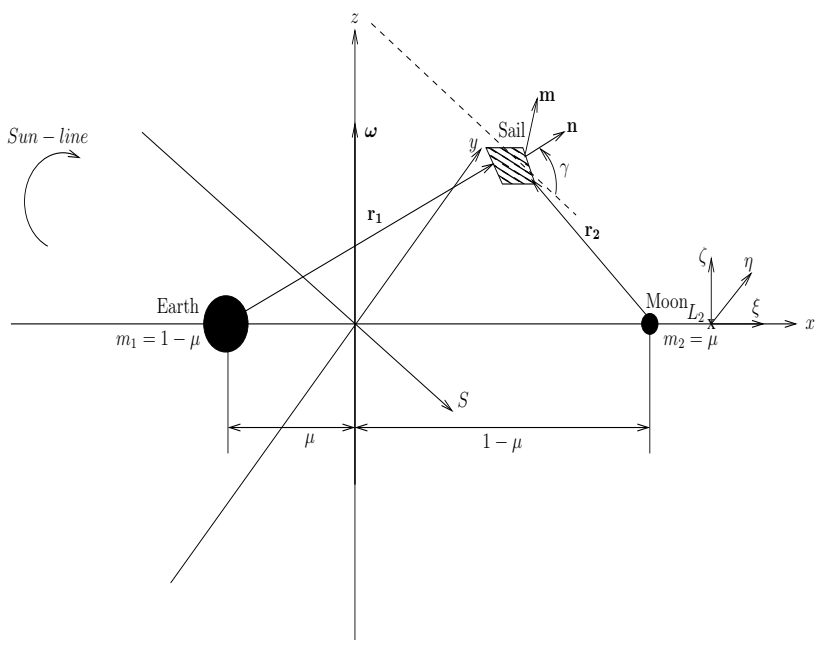

$(a)$

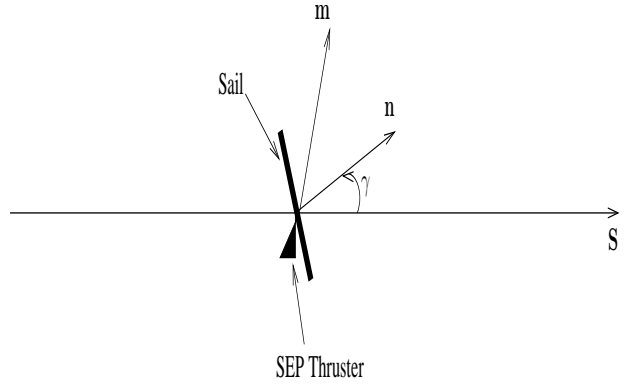

(b)

Figure 1 (a) Schematic geometry of the Hybrid Sail in the Earth-Moon circular restricted three-body problem; $(b)$ Angle $\gamma$ between the Hybrid Sail surface normal $n$ and the Sun-line direction $S$, and SEP thrust vector direction $m$.

the two more massive bodies are moving in circular orbits with constant angular velocity $\omega$ about their common center of mass, and the mass of the third body is too small to affect the motion of the two more massive bodies. The unit mass is taken to be the total mass of the system $\left(m_{1}+m_{2}\right)$ and the unit of length is chosen to be the constant separation $R^{\star}$ between $m_{1}$ and $m_{2}$. The time unit is defined such that $m_{2}$ orbits around $m_{1}$ in time $2 \pi$. Under these considerations the masses of the primaries in the normalized system of units are $m_{1}=1-\mu$ and $m_{2}=\mu$, with $\mu=m_{2} /\left(m_{1}+m_{2}\right)$ (see Figure 1 (a)). The dashed line in Figure 1 (a)) is a line parallel to the Sun-line direction.

\section{Equations of Motions}

The nondimensional equation of a motion of a hybrid sail in the rotating frame of reference is described by

$$
\frac{d^{2} \boldsymbol{r}}{d t^{2}}+2 \boldsymbol{\omega} \times \frac{d \boldsymbol{r}}{d t}+\nabla U(\boldsymbol{r})=\boldsymbol{a}_{S}+\boldsymbol{a}_{S E P}
$$

where $\boldsymbol{\omega}=\omega \hat{\boldsymbol{z}}$ ( $\hat{z}$ is a unit vector pointing in the direction $z$ ) is the angular velocity vector of the rotating frame and $\boldsymbol{r}$ is the position vector of the hybrid sail relative to the center of mass of the two primaries. We will not consider the small annual changes in the inclination of the Sun-line with respect to the plane of the system. The three-body gravitational potential $U(\boldsymbol{r})$, the solar radiation pressure acceleration $\boldsymbol{a}_{S}$ and the nondimensional acceleration due to the SEP thruster $\boldsymbol{a}_{S E P}$ are defined by

$$
\begin{aligned}
U(\boldsymbol{r}) & =-\left[\frac{1}{2}|\boldsymbol{\omega} \times \boldsymbol{r}|^{2}+\frac{1-\mu}{r_{1}}+\frac{\mu}{r_{2}}\right], \\
\boldsymbol{a}_{S} & =a_{0}(\boldsymbol{S} \cdot \boldsymbol{n})^{2} \boldsymbol{n}, \\
\boldsymbol{a}_{S E P} & =a_{S E P} \boldsymbol{m},
\end{aligned}
$$


where $\mu=0.012150582$ is the mass ratio for the Earth-Moon system. The hybrid sail position vectors w.r.t. $m_{1}$ and $m_{2}$ respectively (see Figure 1 (a)), are defined as $\boldsymbol{r}_{1}=[x+\mu, y, z]^{T}$ and $\boldsymbol{r}_{2}=[x-(1-\mu), y, z]^{T}, a_{0}$ is the magnitude of the solar radition pressure acceleration exerted on the hybrid sail and the unit vector $\boldsymbol{n}$ denotes the thrust direction, $\boldsymbol{a}_{S E P}$ is the acceleration from the SEP system and the unit vector $\boldsymbol{m}$ denotes the thrust direction. A constant displacement distance of $1750 \mathrm{~km}$ has been imposed, considering a characteristic acceleration of $a_{0}=0.10 \mathrm{~mm} / \mathrm{s}^{2}$ for the simulations. The sail is oriented such that it is always directed along the Sun-line $S$, pitched at an angle $\gamma$ to provide a constant out-of-plane force. The unit normal to the hybrid sail surface $\boldsymbol{n}$ and the Sun-line direction are given by

$$
\begin{aligned}
\boldsymbol{n} & =\left[\begin{array}{lll}
\cos (\gamma) \cos \left(\omega_{\star} t\right) & -\cos (\gamma) \sin \left(\omega_{\star} t\right) & \sin (\gamma)
\end{array}\right]^{T}, \\
\boldsymbol{S} & =\left[\begin{array}{lll}
\cos \left(\omega_{\star} t\right) & -\sin \left(\omega_{\star} t\right) & 0
\end{array}\right]^{T},
\end{aligned}
$$

where $\omega_{\star}=0.923$ is the angular rate of the Sun-line in the corotating frame in a dimensionless synodic coordinate system.

\section{Linearized System}

We now want to investigate the dynamics of the hybrid sail in the neighborhood of the libration points. We denote the coordinates of the equilibrium point as $\boldsymbol{r}_{L}=\left(x_{L_{i}}, y_{L_{i}}, z_{L_{i}}\right)$ with $i=1, \cdots, 5$. Let a small displacement in $\boldsymbol{r}_{L}$ be $\delta \boldsymbol{r}$ such that $\boldsymbol{r} \rightarrow \boldsymbol{r}_{L}+\delta \boldsymbol{r}$. The equations for the hybrid sail can then be written as

$$
\frac{d^{2} \delta \boldsymbol{r}}{d t^{2}}+2 \boldsymbol{\omega} \times \frac{d \delta \boldsymbol{r}}{d t}+\nabla U\left(\boldsymbol{r}_{L}+\delta \boldsymbol{r}\right)=\boldsymbol{a}_{S}\left(\boldsymbol{r}_{L}+\delta \boldsymbol{r}\right)+\boldsymbol{a}_{S E P}\left(\boldsymbol{r}_{L}+\delta \boldsymbol{r}\right),
$$

and retaining only the first-order term in $\delta \boldsymbol{r}=[\delta x, \delta y, \delta y]^{T}$ in a Taylor-series expansion, the gradient of the potential and the acceleration can be expressed as

$$
\begin{aligned}
\nabla U\left(\boldsymbol{r}_{L}+\delta \boldsymbol{r}\right) & =\nabla U\left(\boldsymbol{r}_{L}\right)+\left.\frac{\partial \nabla U(\boldsymbol{r})}{\partial \boldsymbol{r}}\right|_{\boldsymbol{r}=\boldsymbol{r}_{L}} \delta \boldsymbol{r}+O\left(\delta \boldsymbol{r}^{2}\right), \\
\boldsymbol{a}_{S}\left(\boldsymbol{r}_{L}+\delta \boldsymbol{r}\right) & =\boldsymbol{a}_{S}\left(\boldsymbol{r}_{L}\right)+\left.\frac{\partial \boldsymbol{a}_{S}(\boldsymbol{r})}{\partial \boldsymbol{r}}\right|_{\boldsymbol{r}=\boldsymbol{r}_{L}} \delta \boldsymbol{r}+O\left(\delta \boldsymbol{r}^{2}\right), \\
\boldsymbol{a}_{S E P}\left(\boldsymbol{r}_{L}+\delta \boldsymbol{r}\right) & =\boldsymbol{a}_{S E P}\left(\boldsymbol{r}_{L}\right)+\left.\frac{\partial \boldsymbol{a}_{S E P}(\boldsymbol{r})}{\partial \boldsymbol{r}}\right|_{\boldsymbol{r}=\boldsymbol{r}_{L}} \delta \boldsymbol{r}+O\left(\delta \boldsymbol{r}^{2}\right) .
\end{aligned}
$$

It is assumed that $\nabla U\left(\boldsymbol{r}_{L}\right)=0$, and the accelerations $\boldsymbol{a}_{S}$ and $\boldsymbol{a}_{S E P}$ are constant with respect to the small displacement $\delta \boldsymbol{r}$, so that

$$
\begin{gathered}
\left.\frac{\partial \boldsymbol{a}_{S}(\boldsymbol{r})}{\partial \boldsymbol{r}}\right|_{\boldsymbol{r}=\boldsymbol{r}_{L}}=0, \\
\left.\frac{\partial \boldsymbol{a}_{S E P}(\boldsymbol{r})}{\partial \boldsymbol{r}}\right|_{\boldsymbol{r}=\boldsymbol{r}_{L}}=0 .
\end{gathered}
$$

The linear variational system associated with the libration points at $\boldsymbol{r}_{L}$ can be determined through a Taylor series expansion by substituting Eqs. (5), (6) and (7) into (4) so that

$$
\frac{d^{2} \delta \boldsymbol{r}}{d t^{2}}+2 \boldsymbol{\omega} \times \frac{d \delta \boldsymbol{r}}{d t}-K \delta \boldsymbol{r}=\boldsymbol{a}_{S}\left(\boldsymbol{r}_{L}\right)+\boldsymbol{a}_{S E P}\left(\boldsymbol{r}_{L}\right)
$$


where the matrix $K$ is defined as

$$
K=-\left[\left.\frac{\partial \nabla U(\boldsymbol{r})}{\partial \boldsymbol{r}}\right|_{\boldsymbol{r}=\boldsymbol{r}_{L}}\right] .
$$

Using matrix notation the linearized equation about the libration point (Equation (10)) can be represented by the inhomogeneous linear system $\dot{\mathbf{X}}=A \mathbf{X}+\boldsymbol{b}(t)$, where the state vector $\mathbf{X}=(\delta \boldsymbol{r}, \delta \dot{\boldsymbol{r}})^{T}$, and for which $\boldsymbol{b}(t)($ a $6 \times 1$ vector) is equal to the sum of control accelerations of the sail and the SEP.

The Jacobian matrix $A$ has the general form

$$
A=\left(\begin{array}{cc}
0_{3} & I_{3} \\
K & \Omega
\end{array}\right)
$$

where $I_{3}$ is a identity matrix, and

$$
\Omega=\left(\begin{array}{rrr}
0 & 2 & 0 \\
-2 & 0 & 0 \\
0 & 0 & 0
\end{array}\right)
$$

By making the transformation $\boldsymbol{r} \rightarrow \boldsymbol{r}_{L}+\delta \boldsymbol{r}$ and retaining only the first-order term in $\delta \boldsymbol{r}=$ $(\xi, \eta, \zeta)^{T}$ in a Taylor-series expansion where $(\xi, \eta, \zeta)$ are axes attached to the libration point as shown in Figure 1 (a), the linearized nondimensional equations of motion relative to the collinear libration points can be written as

$$
\begin{aligned}
\ddot{\xi}-2 \dot{\eta}-U_{x x}^{o} \xi & =a_{\xi}+a_{S E P_{\xi}}, \\
\ddot{\eta}+2 \dot{\xi}-U_{y y}^{o} \eta & =a_{\eta}+a_{S E P_{\eta}}, \\
\ddot{\zeta}-U_{z z}^{o} \zeta & =a_{\zeta}+a_{S E P_{\zeta}},
\end{aligned}
$$

where $U_{x x}^{o}, U_{y y}^{o}$, and $U_{z z}^{o}$ are the partial derivatives of the gravitational potential evaluated at the collinear libration point, and the solar sail acceleration is defined in terms of three auxiliary variables $a_{\xi}, a_{\eta}$, and $a_{\zeta}$.

Again, the sail attitude is fixed such that the sail normal vector $\boldsymbol{n}$, which is the unit vector that is perpendicular to the sail surface, points always along the direction of the Sun line with the following constraint $\boldsymbol{S} \cdot \boldsymbol{n} \geq 0$. Its direction is described by the pitch angle $\gamma$ relative to the Sun-line, which represents the sail attitude.

The solar sail acceleration components are therefore given by

$$
\begin{aligned}
& a_{\xi}=a_{0} \cos \left(\omega_{\star} t\right) \cos ^{3}(\gamma), \\
& a_{\eta}=-a_{0} \sin \left(\omega_{\star} t\right) \cos ^{3}(\gamma), \\
& a_{\zeta}=a_{0} \cos ^{2}(\gamma) \sin (\gamma),
\end{aligned}
$$

where $a_{0}$ is the characteristic acceleration. The SEP acceleration components $\boldsymbol{a}_{S E P}$ are used for feedback control as described later. 


\section{TRACKING BY FEEDBACK LINEARIZATION}

Linearization by feedback is a well-known approach to control nonlinear systems. This method transforms a nonlinear state space model into a new coordinate system where the nonlinearities can be cancelled by feedback. It is a way of transforming system models into equivalent models of simpler form.

This technique is completely different from a Jacobian linearization, on which linear control is based. From equation (1) the motion of the hybrid solar sail in the CRTBP is described by the scalar equations in the form

$$
\begin{aligned}
\ddot{\xi} & =2 \dot{\eta}+\left(x_{L_{2}}+\xi\right)-(1-\mu) \frac{\left(x_{L_{2}}+\xi\right)+\mu}{r_{1}^{3}}-\mu \frac{\left(x_{L_{2}}+\xi\right)-1+\mu}{r_{2}^{3}}+a_{\xi}+u_{\xi}, \\
\ddot{\eta} & =-2 \dot{\xi}+\eta-\left(\frac{1-\mu}{r_{1}^{3}}+\frac{\mu}{r_{2}^{3}}\right) \eta+a_{\eta}+u_{\eta}, \\
\ddot{\zeta} & =-\left(\frac{1-\mu}{r_{1}^{3}}+\frac{\mu}{r_{2}^{3}}\right) \zeta+a_{\zeta}+u_{\zeta},
\end{aligned}
$$

where the vector

$$
\boldsymbol{u}(t)=\left[\begin{array}{lll}
u_{\xi} & u_{\eta} & u_{\zeta}
\end{array}\right]^{T}
$$

is the applied control acceleration due to the SEP thruster, such that $\boldsymbol{u}(t) \triangleq \boldsymbol{a}_{S E P}$.

To develop a feedback linearization scheme, the motion of the hybrid solar sail moving in the CRTBP is separated into linear and nonlinear components, such that

$$
\begin{aligned}
& \ddot{\xi}=f_{\text {Non-Linear }}^{\xi}+f_{\text {Linear }}^{\xi}+a_{\xi}+u_{\xi}, \\
& \ddot{\eta}=f_{\text {Non-Linear }}^{\eta}+f_{\text {Linear }}^{\eta}+a_{\eta}+u_{\eta}, \\
& \ddot{\zeta}=f_{\text {Non-Linear }}^{\zeta}+f_{\text {Linear }}^{\zeta}+a_{\zeta}+u_{\zeta},
\end{aligned}
$$

where the $f$ functions are defined as the linear and the nonlinear terms in the equations (20), (21) and (22)

$$
\begin{aligned}
f_{\text {Non-Linear }}^{\xi} & =-(1-\mu) \frac{\left(x_{L_{2}}+\xi\right)+\mu}{r_{1}^{3}}-\mu \frac{\left(x_{L_{2}}+\xi\right)-1+\mu}{r_{2}^{3}} \\
f_{\text {Linear }}^{\xi} & =2 \dot{\eta}+\left(x_{L_{2}}+\xi\right) \\
f_{\text {Non-Linear }}^{\eta} & =-\left(\frac{1-\mu}{r_{1}^{3}}+\frac{\mu}{r_{2}^{3}}\right) \eta \\
f_{\text {Linear }}^{\eta} & =-2 \dot{\xi}+\eta \\
f_{\text {Non-Linear }}^{\zeta} & =-\left(\frac{1-\mu}{r_{1}^{3}}+\frac{\mu}{r_{2}^{3}}\right) \zeta, \\
f_{\text {Linear }}^{\zeta} & =0
\end{aligned}
$$

with $r_{1}=\sqrt{\left(\left(x_{L_{2}}+\xi\right)+\mu\right)^{2}+\eta^{2}+\zeta^{2}} \quad$ and $\quad r_{2}=\sqrt{\left(\left(x_{L_{2}}+\xi\right)-1+\mu\right)^{2}+\eta^{2}+\zeta^{2}}$.

The solar sail acceleration components are given in equations (17), (18) and (19). We then select the SEP control $\boldsymbol{u}(t)$ such that 


$$
\boldsymbol{u}(t)=\left[\begin{array}{l}
u_{\xi} \\
u_{\eta} \\
u_{\zeta}
\end{array}\right]=\boldsymbol{U}(t)+\tilde{\boldsymbol{u}}(t)
$$

where

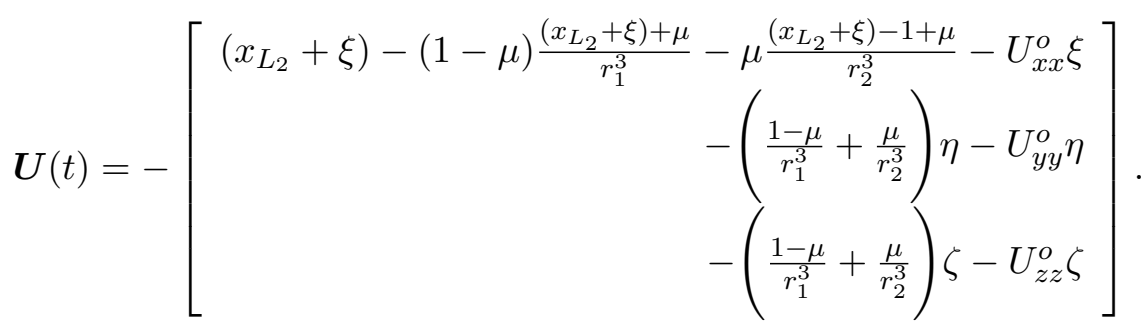

The equations (20), (21) and (22) then become

$$
\begin{aligned}
& \ddot{\xi}=2 \dot{\eta}+U_{x x}^{o} \xi+a_{0} \cos \left(\omega_{\star} t\right) \cos ^{3}(\gamma)+\tilde{u}_{\xi}, \\
& \ddot{\eta}=-2 \dot{\xi}+U_{y y}^{o} \eta-a_{0} \sin \left(\omega_{\star} t\right) \cos ^{3}(\gamma)+\tilde{u}_{\eta}, \\
& \ddot{\zeta}=U_{z z}^{o} \zeta+a_{0} \cos ^{2}(\gamma) \sin (\gamma)+\tilde{u}_{\zeta} .
\end{aligned}
$$

By removing the nonlinear dynamics from the system, the control acceleration vector $\tilde{\boldsymbol{u}}(t)$ is determined such that the desired response characteristics of the linear time-invariant dynamics are produced and so Eq. (35) - (37) are identical to the linear system defined by Eq. (14) - (16). In particular, it can be ensured that the displacement distance of the periodic orbit is constant, which provides key advantages for lunar polar telecommunications.

\section{TRACKING A REFERENCE TRAJECTORY}

\section{Linear Feedback Control}

Let us consider nonlinear system described by

$$
\ddot{\boldsymbol{x}}=f(\boldsymbol{x}, \dot{\boldsymbol{x}})+\boldsymbol{u},
$$

where $\boldsymbol{x} \in \mathbb{R}^{3}$ is the state. Let $\boldsymbol{e}(t)=\boldsymbol{x}(t)-\boldsymbol{x}_{r e f}(t)$ denote the state error relative to some reference solution, where the reference trajectory

$$
\boldsymbol{x}_{r e f}(t)=\left[\begin{array}{lll}
\xi_{\text {ref }} & \eta_{\text {ref }} & \zeta_{\text {ref }}
\end{array}\right]^{T}
$$

is given by the analytical solution

$$
\begin{aligned}
\xi_{\text {ref }}(t) & =\xi_{0} \cos \left(\omega_{\star} t\right), \\
\eta_{\text {ref }}(t) & =\eta_{0} \sin \left(\omega_{\star} t\right), \\
\zeta_{\text {ref }}(t) & =\zeta_{0},
\end{aligned}
$$

which is a solution of the linear equations (14 - 16) with $\boldsymbol{a}_{S E P}=0$ (pure sail at linear order). The term $\boldsymbol{a}_{S E P}$ is only needed to cancel the higher order terms in the expansion. 
We then differentiate $e(t)$ until the control appears so that

$$
\begin{aligned}
\boldsymbol{e}(t) & =\boldsymbol{x}(t)-\boldsymbol{x}_{r e f}(t) \\
\dot{\boldsymbol{e}}(t) & =\dot{\boldsymbol{x}}(t)-\dot{\boldsymbol{x}}_{r e f}(t), \\
\ddot{\boldsymbol{e}}(t) & =\ddot{\boldsymbol{x}}(t)-\ddot{\boldsymbol{x}}_{r e f}(t), \\
& =f(\boldsymbol{x}, \dot{\boldsymbol{x}})+\boldsymbol{u}-\ddot{\boldsymbol{x}}_{r e f}(t), \\
& =-\lambda_{1} \dot{\boldsymbol{e}}-\lambda_{2} \boldsymbol{e},
\end{aligned}
$$

and so, we have

$$
\boldsymbol{u}(t)=-f(\boldsymbol{x}, \dot{\boldsymbol{x}})+\ddot{\boldsymbol{x}}_{r e f}(t)-\lambda_{1} \dot{\boldsymbol{e}}-\lambda_{2} \boldsymbol{e},
$$

where $-\lambda_{1} \dot{e}-\lambda_{2} e$ is the stabilizing term.

\section{Trajectory Tracking}

Consider the system given by (38), where our objective is to make the output $\boldsymbol{x} \in \mathbb{R}^{3}$ track a desired trajectory given by the reference trajectory $\boldsymbol{x}_{r e f} \in \mathbb{R}^{3}$ while keeping the position bounded. Therefore, we want to find a control law for the input $\tilde{\boldsymbol{u}} \in \mathbb{R}^{3}$ such that, starting from any initial state in a domain $D \subset \mathbb{R}^{3}$, the tracking error $\boldsymbol{e}(t)=\boldsymbol{x}(t)-\boldsymbol{x}_{r e f}(t)$ goes to zero.

Hence, asymptotic tracking will be achieved if we design a state feedback control law to ensure that $\boldsymbol{e}(t)$ is bounded and converges to zero as $t$ tends to infinity.

Thus, the control law

$$
\tilde{\boldsymbol{u}}=-\lambda_{1} \dot{\boldsymbol{e}}-\lambda_{2} \boldsymbol{e}
$$

yields the tracking error equation

$$
\ddot{e}+\lambda_{1} \dot{e}+\lambda_{2} \boldsymbol{e}=0
$$

where $\lambda_{1}$ and $\lambda_{2}$ are chosen positive constants.

\section{EVALUATION OF HYBRID SAIL PERFORMANCE}

In this section we investigate the performance of the hybrid sail system, constituted by a solar sail combined with solar electric propulsion. The simulation was performed around the collinear libration point $L_{2}$ for a period of one month. The magnitude of the total control effort appears in Figure 4. Thus, the control acceleration effort $\boldsymbol{U}(t)$ required to track the reference orbit while rejecting the nonlinearities varies up to $0.005\left(0.014 \mathrm{~mm} / \mathrm{s}^{2}\right)$ about the $L_{2}$ point. The control accelerations are continous smooth signals. The acceleration derived from the solar sail (denoted by $a_{\xi}, a_{\eta}, a_{\zeta}$ ) is plotted in terms of components for one-month orbits in Figure 2 (a) about $L_{2}$, and the SEP acceleration components appears in Figure 2 (b) about $L_{2}$. The control acceleration effort derived from the thruster (denoted by $U_{\xi}, U_{\eta}, U_{\zeta}$ ) is order of $10^{-3}-10^{-4}$, while the acceleration derived from the solar sail is approximately $10^{-2}$. The small control acceleration from the SEP thruster is then applied to ensure that the displacement of the periodic orbit is constant. The solar sail provides a constant out-of-plane force.

Figure 3 (a) (resp. Figure 3 (b)) illustrates the position error components, denoted by $e_{\xi}, e_{\eta}, e_{\zeta}$ (resp. velocity error components, denoted by $e_{\xi d}, e_{\eta d}, e_{\zeta d}$ ) under the nonlinear control and the SEP thruster around $L_{2}$. These Figures show that the motion is bounded and periodic. This observation implies that the augmented thrust acceleration ensures a constant displacement orbit. 

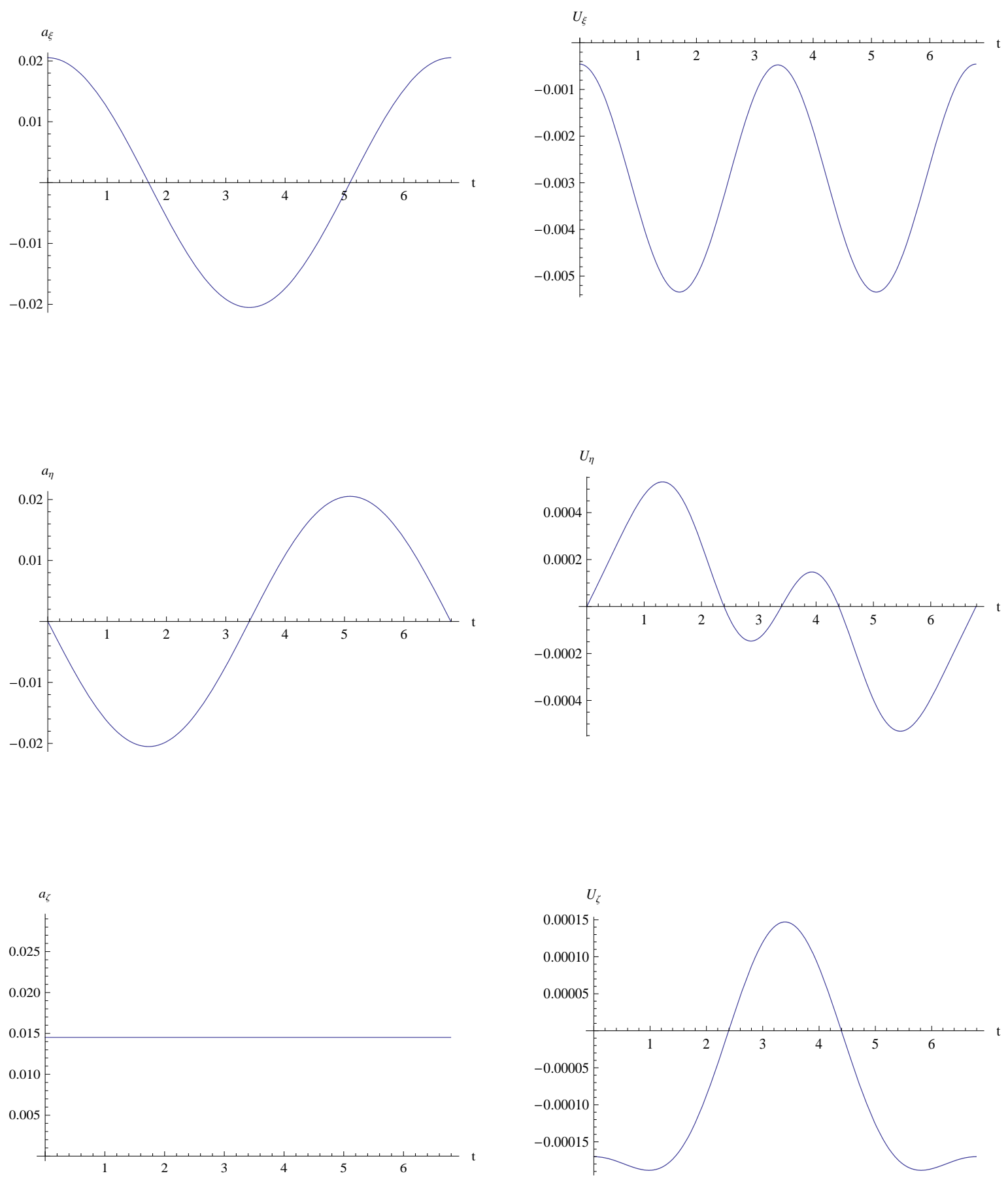

(a)

(b)

Figure 2 (a) Acceleration derived from the solar sail about the $L_{2}$ point; $(b)$ Acceleration derived from the SEP thruster about the $L_{2}$ point. 

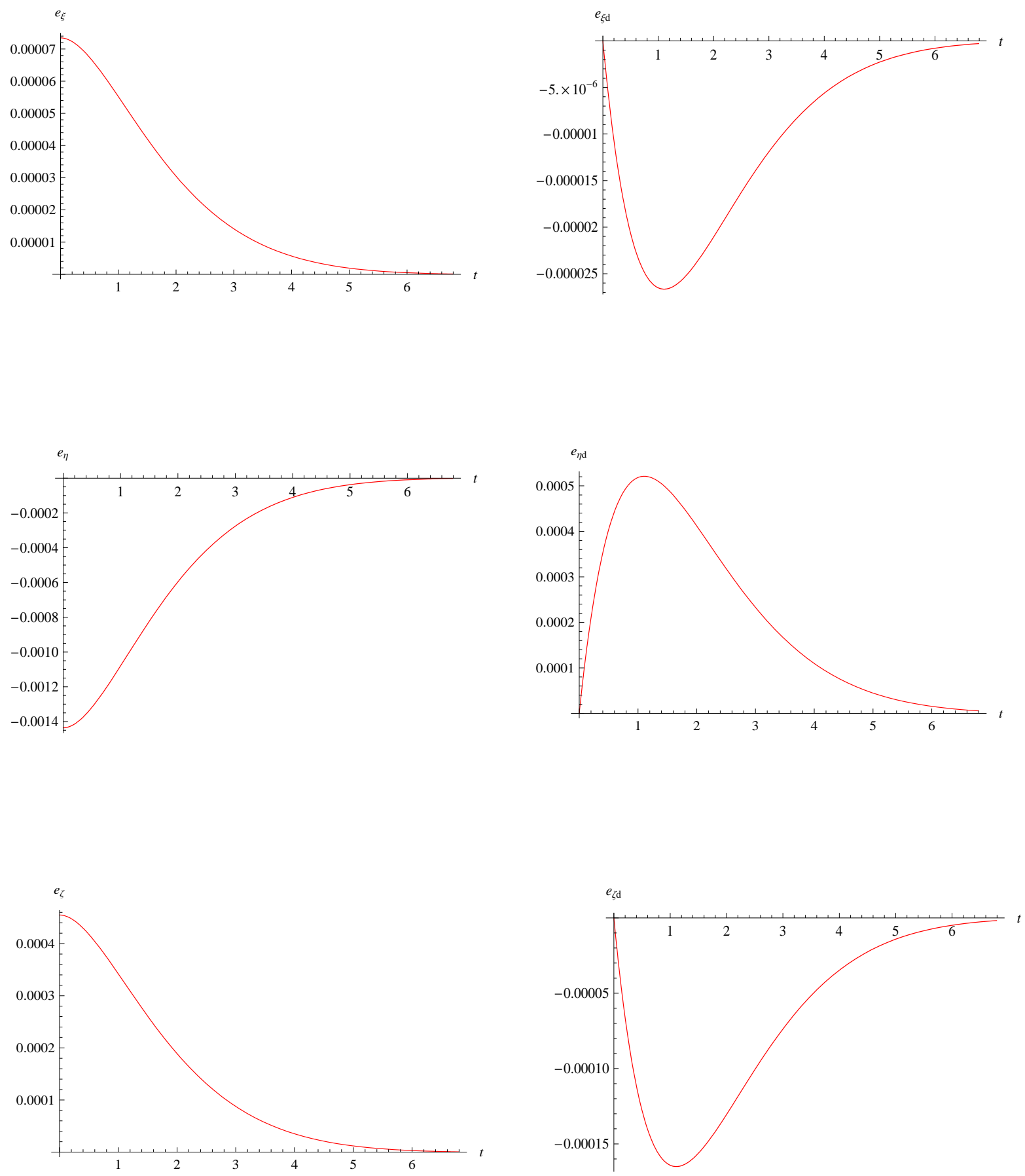

(a)

(b)

Figure $3(a)$ Position error components with $e(0)=(0.000073,-0.0014,0.00045)^{T}$; (b) Velocity error components. 


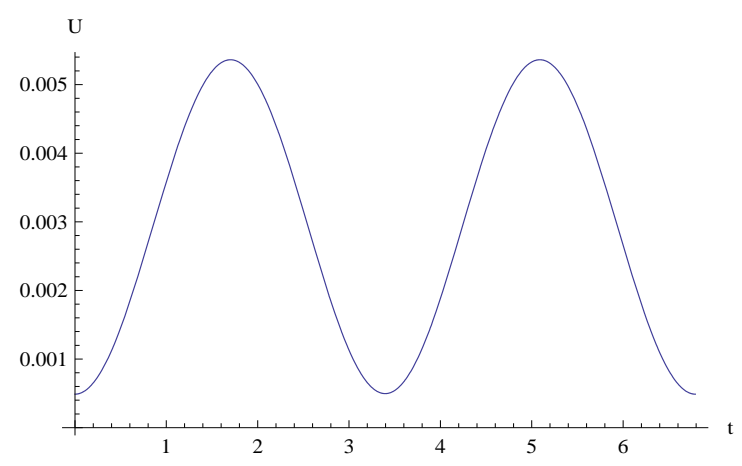

Figure 4. Magnitude of the total control effort about the $L_{2}$ point.

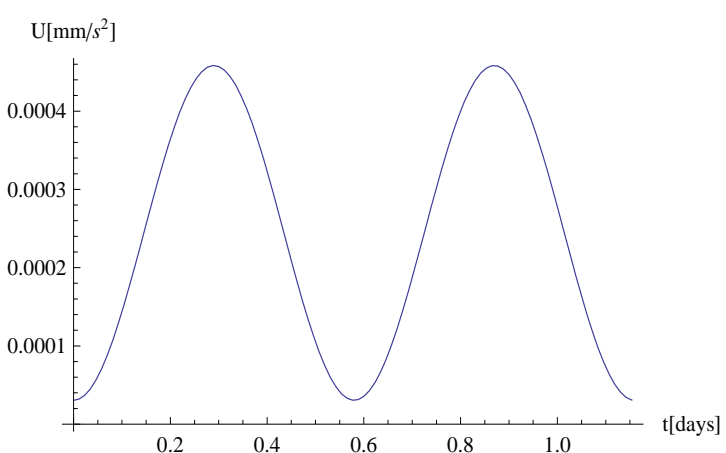

(a)

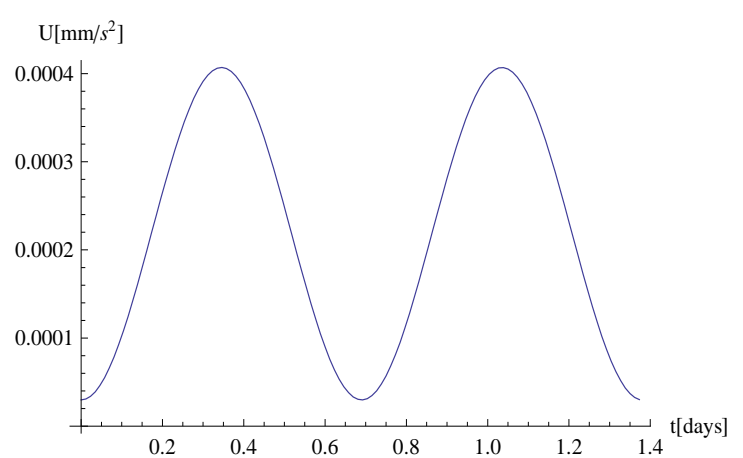

(b)

Figure 5 (a) Magnitude of the total control effort, $\mu=0.25$; (b) Magnitude of the total control effort, $\mu=0.5$.

\section{APPLICATIONS TO BINARY ASTEROID SYSTEMS}

The previous section established a hybrid concept for displaced lunar orbits, using the EarthMoon System as the primaries in the circular restricted three-body problem. The application to be treated here is related to binary asteroid systems. Thus, several set of curves of the control acceleration effort required to track a reference orbit while rejecting the nonlinearities are shown from $\mu=0.15$ to $\mu=0.5$.

The magnitude of the total control effort appears in Figure 5 (a) for system mass ratio $\mu=0.25$, and Figure 5 (b) for $\mu=0.5$. The control acceleration effort $\boldsymbol{U}(t)$ required to track the reference orbit while rejecting the nonlinearities varies up to $0.0004 \mathrm{~mm} / \mathrm{s}^{2}$ for $\mu=0.25$ and $\mu=0.5$. Again, the control accelerations are continous smooth signals. The acceleration derived from the solar sail (denoted by $a_{\xi}, a_{\eta}, a_{\zeta}$ ) is plotted in terms of components for one revolution of the asteroid orbit in Figure 6 (a), and the SEP acceleration components appears in Figure 6 (b) for system mass ratio $\mu=0.25$. Similarly, the acceleration derived from the solar sail is plotted in terms of components for one revolution of the asteroid orbit in Figure 7 (a), and the SEP acceleration components appears in Figure 7 (b) for system mass ratio $\mu=0.5$. The control acceleration effort derived from the thruster (denoted by $U_{\xi}, U_{\eta}, U_{\zeta}$ ) is order of $10^{-4}-10^{-5}$, while the acceleration derived from the solar sail is approximately $10^{-2}$ for $\mu=0.25$ and $\mu=0.5$.

The numerical results indicate that these conclusions might be extended up to $\mu=0.5$. The 
practical importance of such trajectories is due to the fact that the sensitivity to errors in guidance is small. 

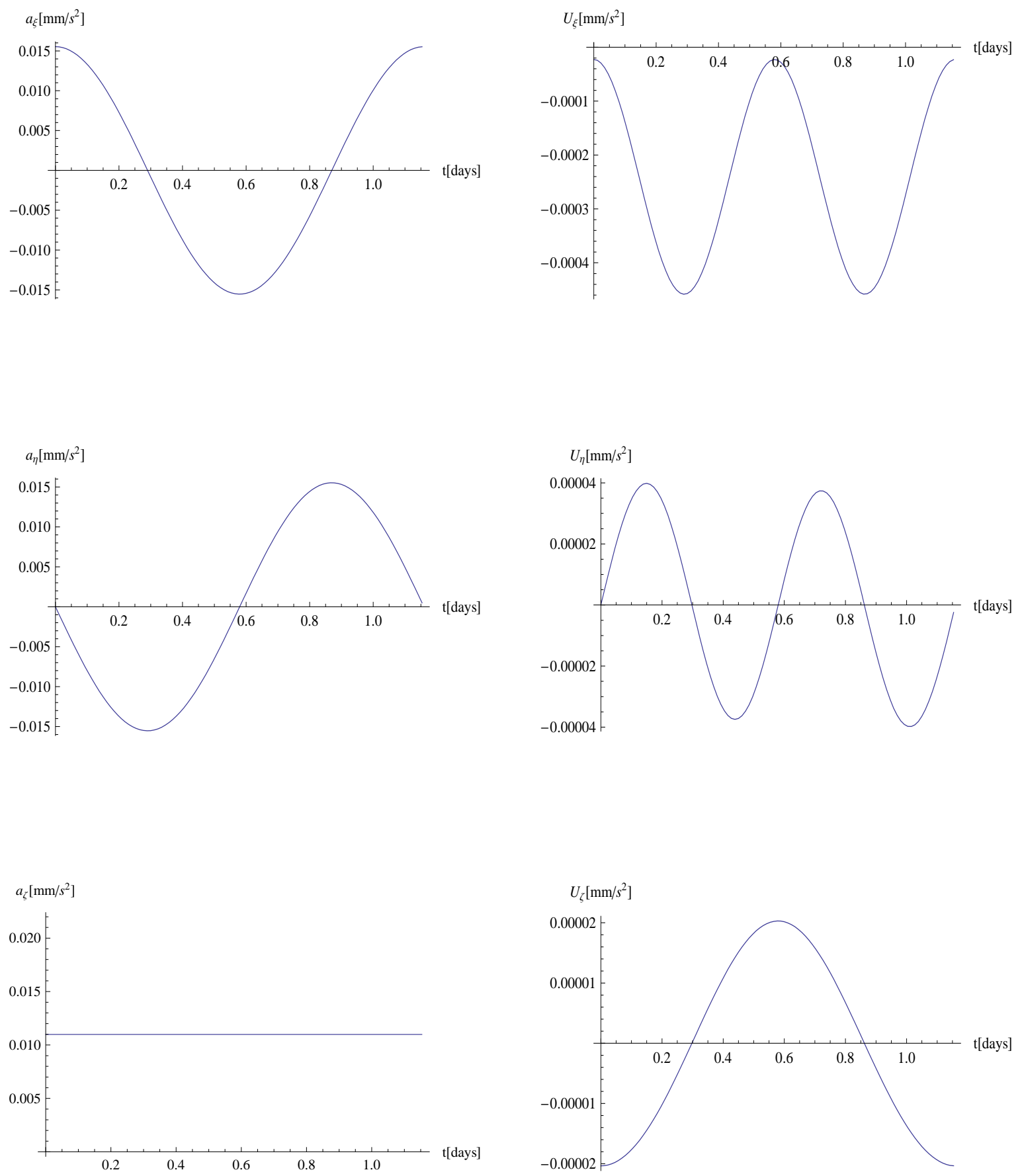

(a)

(b)

Figure 6 (a) Acceleration derived from the solar sail with the system mass ratio $\mu=0.25 ;(b)$ Acceleration derived from the SEP thruster with the system mass ratio $\mu=0.25$. 

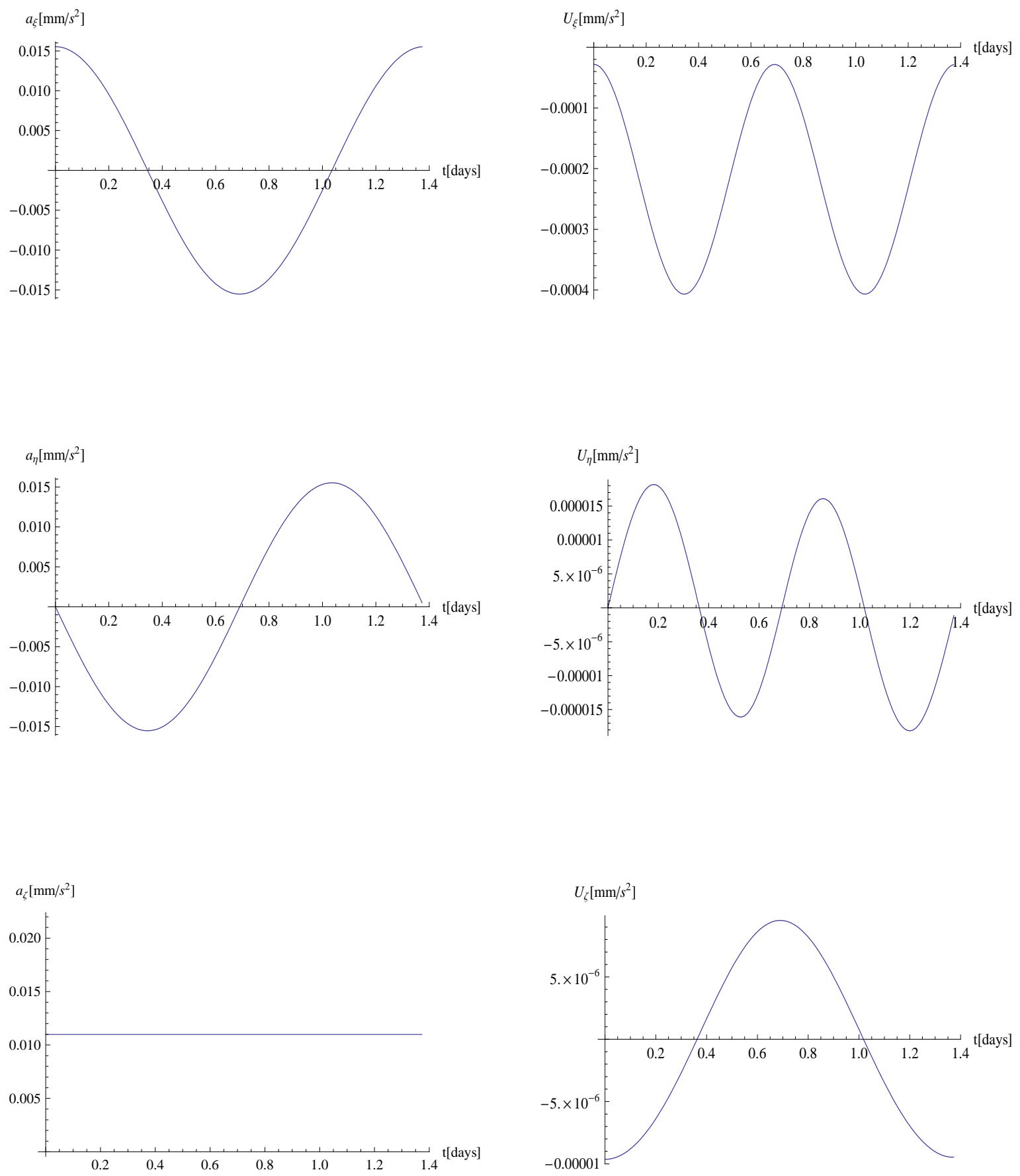

(a)

(b)

Figure $7 \quad(a)$ Acceleration derived from the solar sail with the system mass ratio $\mu=0.5$; (b) Acceleration derived from the SEP thruster with the system mass ratio $\mu=0.5$. 


\section{CONCLUSIONS}

Using the Earth-Moon System as the primaries in the circular restricted three-body problem, a hybrid concept for displaced lunar orbits has been developed. A feedback linearization was used to perform stabilization and trajectory tracking for the nonlinear system. The idea of this control is to transform a given nonlinear system into a linear system by use of a nonlinear coordinate transformation and nonlinear feedback. The augmented thrust acceleration is than applied to ensure a constant displacement periodic orbit, which provides key advantages for lunar polar telecommunications. A stabilizing approach is then introduced to increase the damping in the system and to allow a higher gain in the controller.

\section{REFERENCES}

[1] J. Simo and C. R. McInnes, "Stabilization of Displaced Periodic Orbits in the Solar Sail Restricted Three-Body Problem," presented at the SIAM Conference on Applications of Dynamical Systems (DS09), Snowbird, Utah, May 17 - 21, 2009.

[2] J. Simo and C. R. McInnes, "Asymptotic Analysis of Displaced Lunar Orbits," Journal of Guidance, Control and Dynamics, Vol. 32, No. 5, September-October 2009, pp. 1666-1671.

[3] J. Simo and C. R. McInnes, "Analysis and Control of Displaced Periodic Orbits in the Earth-Moon System," In $60^{\text {th }}$ International Astronautical Congress, Daejeon, Republic of Korea, 12 - 16 October 2009. IAC-09.C1.2.4.

[4] J. Simo and C. R. McInnes, "Displaced Periodic Orbits with Low-Thrust Propulsion in the Earth-Moon System," In $19^{\text {th }}$ AAS/AIAA Space Flight Mechanics Meeting, Savannah, Georgia, February 8 - 12 , 2009. AAS 09-153.

[5] J. Simo and C. R. McInnes, "Designing Displaced Lunar Orbits Using Low-Thrust Propulsion," Journal of Guidance, Control and Dynamics, Vol. 33, No. 1, January-February 2010.

[6] J. Simo and C. R. McInnes, "Displaced solar sail orbits: Dynamics and applications," In 20"h AAS/AIAA Space Flight Mechanics Meeting, San Diego, California, February 14-17, 2010. AAS 10-222.

[7] J. Simo and C. R. McInnes, "Tracking Unstable Periodic Orbits in the Circular Restricted Three-Body Problem," Workshop on Applications on Control theory to Astrodynamics problems, Surrey, England, April 26-27, 2010.

[8] M. Leipold and M. Götz, "Hybrid Photonic/Electric Propulsion," Kayser-Threde, TR SOL4- TR-KTH0001, Munich, Jan. 2002, ESA Contract No. 15334/01/NL/PA.

[9] G. Mengali and A. A. Quarta, "Trajectory Design with Hybrid Low-Thrust Propulsion system," Journal of Guidance, Control, and Dynamics, Vol. 30, No. 2, March-April 2007, pp. 419-426.

[10] B. Dachwald, W. Seboldt, and B. Häusler, "Performance Requirements for Near-Term Interplanetary Solar Sailcraft Missions," $6^{\text {th }}$ International Symposium on Propulsion for Space Transportation of the XXIst Century, Versailles, France, May 2002.

[11] C. R. McInnes, Solar sailing: technology, dynamics and mission applications. London: Springer Praxis, 1999.

[12] T. Waters and C. McInnes, "Periodic Orbits Above the Ecliptic in the Solar-Sail Restricted Three-Body Problem," Journal of Guidance, Control, and Dynamics, Vol. 30, No. 3, 2007, pp. 687-693.

[13] J. Simo and C. R. McInnes, "Solar sail trajectories at the Earth-Moon Lagrange points," In $59^{\text {th }}$ International Astronautical Congress, Glasgow, Scotland, 29 Sep - 03 Oct 2008. Paper IAC-08.C1.3.13.

[14] J. Simo and C. R. McInnes, "Solar Sail Orbits at the Earth-Moon Libration points," Communications in Nonlinear Science and Numerical Simulation, Vol. 14, No. 12, December 2009, pp. 4191-4196.

[15] R. J. McKay, M. Macdonald, M. Vasile, and F. B. d. Frescheville, "A Novel Interplanetary Communications Relay," In AIAA/AAS Astrodynamics Specialist Conference and Exhibit, Toronto, Canada, August 2010. Paper AIAA-2010-7964.

[16] M. Ozimek, D. Grebow, and K. Howell, "Solar Sails and Lunar South Pole Coverage," In AIAA/AAS Astrodynamics Specialist Conference and Exhibit, Honolulu, Hawaii,August 2008. Paper AIAA-20087080.

[17] G. G. Wawrzyniak and K. Howell, "Numerical Techniques for Generating and Refining Solar Sail Trajectories," Advances in Space Research, Vol. 48, 2011.

[18] M. Ceriotti and C. McInnes, "Generation of Optimal Trajectories for Earth Hybrid Pole-Sitters," Journal of Guidance, Control, and Dynamics, Vol. 34, No. 3, May-June 2011, pp. 847-859. 
[19] J. Heiligers, M. Ceriotti, C. Mcinnes, and J. Biggs, "Displaced Geostationary Orbit Design Using Hybrid Sail Propulsion," Journal of Guidance, Control, and Dynamics, Vol. 34, No. 6, NovemberDecember 2011, pp. 1852-1866.

[20] V. Szebehely, Theory of Orbits: the restricted problem of three bodies. New York and London: Academic Press, 1967.

[21] R. Farquhar and A. Kamel, "Quasi-periodic orbits about the translunar libration point," Celestial Mechanics, Vol. 7, 1973, pp. 458-473.

[22] F. Vonbun, "”A Humminbird for the L 2 Lunar Libration Point"," Nasa TN-D-4468, April 1968.

[23] G. Gómez, A. Jorba., J.Masdemont, and C. Simó, Dynamics and Mission Design Near Libration Points, Vol. III, IV. Singapore.New Jersey.London.Hong Kong: World Scientific Publishing Co.Pte.Ltd, 2001.

[24] J. Breakwell and J. Brown, "The 'halo' family of 3-dimensional periodic orbits in the Earth-Moon restricted 3-body problem,' Celestial Mechanics, Vol. 20, 1979, pp. 389-404.

[25] K. Howell, “Three-dimensional, periodic, 'halo' orbits,” Celestial Mechanics, Vol. 32, 1984, pp. 53-71.

[26] K. Howell and B. Marchand, “"Natural and Non-Natural Spacecraft Formations Near $\mathrm{L}_{1}$ and $\mathrm{L}_{2}$ Libration Points in the Sun-Earth/Moon Ephemerics System"," Dynamical Systems: An International Journal, Vol. 20, No. 1, March 2005, pp. 149-173.

[27] J.-J. E. Slotine and W. Li, Applied Nonlinear Control. Englewood Cliffs, New Jersey 07632: Prentice Hall, 1991.

[28] J. Bellerose and D. J. Scheeres, "Mission to Binary Asteroids: 1999 KW4 as a Case Study," In $18^{\text {th }}$ AAS/AIAA Space Flight Mechanics Meeting, Galveston, Texas, January 27 - 31, 2008. Paper AAS 08170.

[29] D. Scheeres and R. Schweickart, "The Mechanics of Moving Asteroids," $1^{\text {st }}$ Planetary Defense Conference, Orange County, California, February 23 - 26, 2004. AIAA 2004-1446.

[30] S. B. Broschart and D. J. Scheeres, "Control of Hovering Spacecraft Near Small Bodies: Application to Asteroid 25143 Itokawa," Journal of Guidance, Control, and Dynamics, Vol. 28, No. 2, March-April 2005, pp. 343-354.

[31] J. P. Sanchez and C. R. McInnes, “Asteroid Resource Map for Near-Earth Space,” Journal of Spacecraft and Rocket, Vol. 48, No. 1, January-February 2011, pp. 153-165.

[32] H. Baoyin and C. McInnes, "Solar sail halo orbits at the Sun-Earth artificial L ${ }_{1}$ point," Celestial Mechanics and Dynamical Astronomy, Vol. 94, No. 2, 2006, pp. 155-171.

[33] S. Baig and C. McInnes, "Artificial Three-Body Equilibria for Hybrid Low-Thrust Propulsion," Journal of Guidance, Control, and Dynamics, Vol. 31, No. 6, November-December 2008, pp. 1644-1655. 